\title{
From String Nets to Nonabelions
}

\author{
Lukasz Fidkowski ${ }^{1,4}$, Michael Freedman ${ }^{1}$, Chetan Nayak $^{1,2}$, \\ Kevin Walker ${ }^{1}$, Zhenghan Wang ${ }^{1,3}$ \\ 1 Microsoft Station Q, University of California, Santa Barbara 93106-6105, USA. \\ E-mail: zhenghwa@microsoft.com \\ 2 Department of Physics and Astronomy, University of California, Los Angeles, CA 90095-1547, USA \\ 3 Department of Mathematics, Indiana University, Bloomington, IN 47405, USA \\ 4 Department of Physics, Stanford University, Stanford, CA 94305, USA
}

Received: 23 January 2007 / Accepted: 12 December 2008

Published online: 14 February 2009 - (C) The Author(s) 2009. This article is published with open access at Springerlink.com

\begin{abstract}
We discuss Hilbert spaces spanned by the set of string nets, i.e. trivalent graphs, on a lattice. We suggest some routes by which such a Hilbert space could be the low-energy subspace of a model of quantum spins on a lattice with short-ranged interactions. We then explain conditions which a Hamiltonian acting on this string net Hilbert space must satisfy in order for the system to be in the DFib (Doubled Fibonacci) topological phase, that is, be described at low energy by an $S O(3)_{3} \times S O(3)_{3}$ doubled Chern-Simons theory, with the appropriate non-abelian statistics governing the braiding of the low-lying quasiparticle excitations (nonabelions). Using the string net wavefunction, we describe the properties of this phase. Our discussion is informed by mappings of string net wavefunctions to the chromatic polynomial and the Potts model.
\end{abstract}

\section{Introduction}

In two dimensions, exotic quantum systems exist where interchange of identical quasiparticle excitations (often called anyons) can alter the wavefunction by a phase not equal to \pm 1 (as in the case of bosons and fermions). In fact, even non-abelian matrix operations, rather than just phases, are possible. The mathematical theory of anyons modular tensor categories - is extremely rich in examples, and existing physical theory provides a way of describing most of these examples as effective Chern-Simons gauge theories. In contrast, our knowledge is limited when it comes to identifying plausible solid state Hamiltonians from which a (2D) state of matter could emerge whose effective low energy description is, in fact, a Chern-Simons theory. The off-diagonal conductivity of fractional quantum Hall (FQHE) systems is tantamount to the equation of motion for a Chern-Simons Lagrangian, so Hall systems are the most developed source of such examples. The best-studied example among abelian states is the $v=1 / 3$ Laughlin state. The foremost candidate among non-Abelian states is the Pfaffian state [3], which is believed $[4,5]$ to be realized at the $v=5 / 2$ fractional quantum hall plateau. Beyond 
$v=5 / 2$, more delicate plateaus at $v=12 / 5,4 / 7$, etc. may also support nonabelions. However, in this paper we explore a quite distinct family of Hamiltonians.

Because magnetic interactions in solids can be at energy scales as high as $\sim 10^{3}$ Kelvin, it would be very exciting to find realistic families of spin Hamiltonians representing a nonabelian phase. (This has essentially been accomplished [6] for the simplest abelian phase, $Z_{2}$ gauge theory, although the corresponding experimental system has not been clearly identified.) This goal has been pursued for several years through the study of model Hamiltonians $H$ acting in an effective Hilbert space $\mathcal{H}$ whose degrees of freedom are either unoriented loops [7], or, more recently, branching loops called "string nets" [1]. Such Hilbert spaces $\mathcal{H}$ are a kind of half-way house. Eventually, it will be necessary to understand how local spins can encode effective loops and nets, and some ideas on encoding nets are presented in Sect. 2. However, the premise of this paper is that we already have a Hilbert space $\mathcal{H}$ spanned by the simplest type of string net $G$, where the lines are unoriented and unlabeled, the nodes have valence 3 and lack internal states. Our goal then is to formulate, in the most general terms, what properties a Hamiltonian $H: \mathcal{H} \rightarrow \mathcal{H}$ should have in order to describe the simplest topological phase of string nets, the "doubled Fibonacci theory", DFib, also sometimes denoted $S O(3)_{3} \times S O(3)_{3}$. DFib is not only nonabelian but its braiding statistics is sufficiently rich as to serve as a basis for universal quantum computation [13]. It is thus an extremely attractive target phase.

Following the microscopics of Sect. 2, we proceed in Sect. 3 to a derivation of DFib based on the concept of minimal degeneracy. To summarize our approach in a phrase: "nature abhors a degeneracy". (Consider, for example, eigenvalue repulsion for random Hamiltonians). There is an irony here because topological phases are nothing else than a degenerate, yet stable, ground state for which no classical symmetry exists to be broken. From this viewpoint, we will see that building DFib (and other phases?) amounts to setting a trap for nature. By compelling a certain space $V(D, n)$ of low energy modes (see Sec. 3 for a precise definition of $V(D, n)$ ) for small $n$ to have dimension equal to $d$, unexpectedly small, we trap a class of Hamiltonians into an exponential growth of degeneracy: $\lim _{n \rightarrow \infty}(\operatorname{dim}(V(D, n)))^{1 / n}=\tau=\frac{1+\sqrt{5}}{2}$. We present a rather surprising derivation of DFib from dimensional considerations alone; the $F$-matrix (or $6 j$ symbol) derives from the assumption of unitarity and minimal dimension of "disk spaces". Although the $F$-matrix obeys the pentagon equations we do not use the pentagon equation to find the $F$-matrix. The physical significance is that DFib should be a robust phase stabilized by a type of eigenvalue repulsion.

Section 4 treats quasiparticle excitations. After DFib is derived in Sect. 5, a beautiful formula of Tutte (compare Ref. [2]) allows us to make an exact connection to the $Q=\tau+2 \approx 3.618$ state Potts model, where $\tau=\frac{1+\sqrt{5}}{2}$, the golden ratio. We find that the exactly solvable point in the DFib phase is the high temperature limit of the low temperature expansion of the $\tau+2$-state Potts model. This allows us to conjecture that the topological phase extends downward in "temperature" until the critical point is reached at $\log \beta=\sqrt{\tau+2}+1$. This suggests a one-parameter family of DFib-Hamiltonians whose ground state wave functions are not strictly topological but have a "length" or bond-fugacity, $x$, satisfying $0.345 \approx 1 /(\sqrt{\tau+2}+1) \leq x \leq 1$, implying a considerable stability within this phase.

The approach in Sect. 5 and 6 complements the ideas presented in [1 and 2]. In [1], a finely tuned exactly solvable fixed point for DFib was produced; in this paper we focus instead on the minimum general requirements for the phase. In Sect. 5 we find an identity which is used in Sect. 6 to connect the statistical physics of DFib's ground states to a critical Potts model, validating a key argument of [2]. 
To recap, the paper is organized as follows. In Sect. 2, some ideas are presented for how string nets could emerge from microscopic models of quantum spins on a lattice. In Sect. 3, DFib is derived from dimensional considerations. In Sect. 4, we describe, in string net language, the quasiparticle excitations of DFib. In Sect. 5, string net wavefunctions and their squares are related to the chromatic polynomial. In Sect. 6 we show that the topological string net wave function has a "plasma analogy" to the $\tau+2$-state Potts model, and in Sect. 7 we discuss our conclusions. In the Appendix, Baxter's hard hexagon model is used to extend a theorem of Tutte's.

\section{How to Construct a Net Hilbert Space $\mathcal{H}$}

In this paper, we will be concerned with Hamiltonians $H$ acting on Hilbert spaces $\mathcal{H}(\Sigma)$ of wave functions $\Psi$ that assign complex-valued amplitudes to string nets ("nets" for short) on a surface $\Sigma$. The surfaces $\Sigma$ which could be relevant to experimental systems are presumably planes with some number of punctures. However, it is quite profitable conceptually and for the purpose of numerical simulations to think about higher-genus surfaces as well. A net is what mathematicians call a trivalent graph; it has only simple branching and no "dead ends" (univalent vertices) except as defined by boundary conditions at the edge of the surface. According to this definition, (the Dirac function on the net in) Fig. 1 a) is not in the Hilbert space $\mathcal{H}$ but (those of) Fig. 1 b) and 1 c) are. In most of this paper, we will simply assume that the Hilbert spaces $\mathcal{H}(\Sigma)$ arise as the low-energy subspaces of the Hilbert spaces of a system of spins or electrons in a solid or ultra-cold atoms on an optical lattice. In such a formulation Fig. 1 a) could be thought of as a high, but finite, energy state of the spins, electrons in a solid or ultra-cold atoms.

In this section, however, we will consider the question: from what kinds of lattice models do nets emerge in the low-energy description so that the Hilbert spaces $\mathcal{H}(\Sigma)$ are the low-energy subspaces? Three ideas A, B, and C are sketched for writing a spin Hamiltonian $K: \bar{H} \rightarrow \bar{H}$ on a large Hilbert space $\bar{H}$ of microscopic degrees of freedom so that the ground state manifold $\mathcal{H}$ of $K$ will be the "Hilbert space of nets" on which this paper is predicated. We emphasize that these spin Hamiltonians only reduce the Hilbert space down to a still rather large one supported on nets; they are not the full Hamiltonians describing the topological phase. In particular, their generic ground state wave functions do not satisfy isotopy invariance. We believe that they could be made to do so with the addition of extra terms to the hamiltonian, and this is the approach we take: first, cut the degrees of freedom to string nets, then make the string nets fluctuate appropriately to gain isotopy invariance. In the following, we accomplish the first part of this process.

(a)

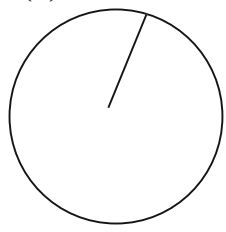

(b)

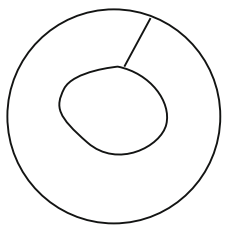

(c)

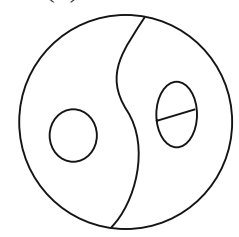

Fig. 1. a) is not in the Hilbert space $\mathcal{H}$, but b) and c) are. In these figures, the outermost circle is the boundary of the system, where nets are allowed to terminate. The endpoint in the middle in (a) is a violation of the "no dead ends" condition 


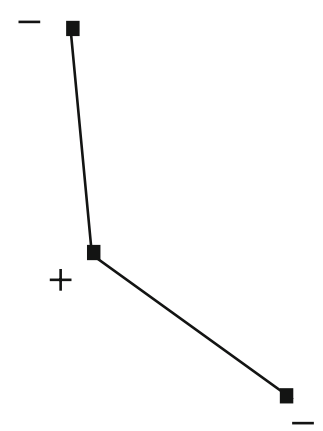

Fig. 2. An illegal pair of bonds which is energetically penalized by $K_{p}$

All our Hamiltonians $K$ break $S U(2)$-invariance and require fine tuning. Ideas A and $\mathrm{B}$ are conceptually very simple but both require a three-body interaction. Idea $\mathrm{C}$ is really an encryption of B into a 2-body interaction on higher spin $(\operatorname{spin}=3 / 2)$ particles.

A. $\bar{H}=\otimes_{\text {bonds }} C^{2}$, i.e. is a Hilbert space of spin $=1 / 2$ particles living on the links of a trivalent graph such as the honeycomb. We interpret an $S_{i}^{z}=1 / 2$ link as one on which the net lies. We take $K=\Sigma_{\text {sites }} K_{S}$ where $K_{S}$ projects onto the subspace of $C^{2} \otimes C^{2} \otimes C^{2}$ (i.e. of the Hilbert space of the three spins surrounding a lattice) of total $\sum_{i=1}^{3} S_{i}^{z}=-1 / 2$. This forbids dead ends. Unfortunately, the function

$$
\text { total } \begin{aligned}
S^{z} \text { eigenvalue } & \rightarrow \text { energy } \\
-3 / 2 & \rightarrow 0, \\
-1 / 2 & \rightarrow \text { nonzero } \\
1 / 2 & \rightarrow 0 \\
3 / 2 & \rightarrow 0
\end{aligned}
$$

is not quadratic (no parabola passes through $(-3 / 2,0),(-1 / 2$, nonzero), $(1 / 2,0)$, and $(3 / 2,0)$. Hence, when $K_{s}$ is expanded in products of $\sigma^{z}{ }_{i}, i=1,2,3$, running over the bonds meeting $s$, it must contain a cubic $\sigma^{z}{ }_{1} \sigma_{2} \sigma^{z}{ }_{3}$ term.

$B$. An alternative is to put a spin $=1 / 2$ particle at the sites of the honeycomb, so $\mathcal{H}=\otimes_{\text {sites }} C^{2}, C^{2}=\langle+,-\rangle$. For each consecutive pair $p$ of bonds, $K$ has a term $K_{p}$ : $K=\Sigma_{p} K_{p}$, where $K_{p}$ is a diagonal matrix all of whose entries are 0 except for that corresponding to the illegal pair of edges shown in Fig. 2). $K$ penalizes both isolated +'s and +'s with exactly one + neighbor. The latter situation is shown in Fig. 3. Interpreting those bonds bounded by two + signs as the ones on which the net lies, we see that the zero modes of $K$ are precisely the nets (trivalent graphs) within the honeycomb. Unfortunately $K$ seems resolutely 3-body.

$B^{\prime}$. To set the stage for our final construction, it is helpful to reverse + and - spins on the index 2 Bravais lattice $L^{\prime}$ within the honeycomb, honeycomb $=L \bigcup L^{\prime}$. With this

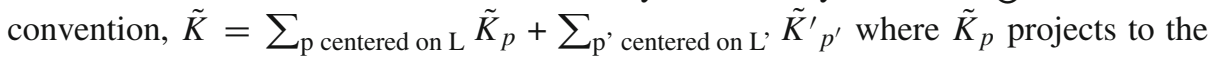
highest total $S_{z}$ eigenvalue, $S_{z}=3 / 2$, and $\tilde{K}_{p}^{\prime} p^{\prime}$ projects to the lowest total $S_{z}$ eigenvalue, $S_{z}=-3 / 2$. In other words, the penalized configurations are 3 consecutive pluses or 3 consecutive minuses. Also, we now draw bonds between plus sites of $L$ and minus sites 


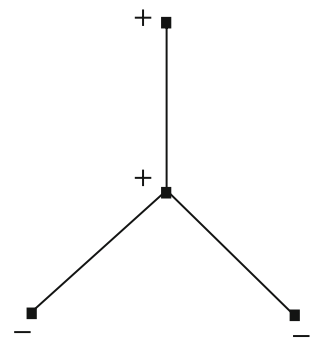

Fig. 3. An up-spin which has only a single up-spin neighbor. This is energetically penalized by $K=\Sigma_{p} K_{p}$

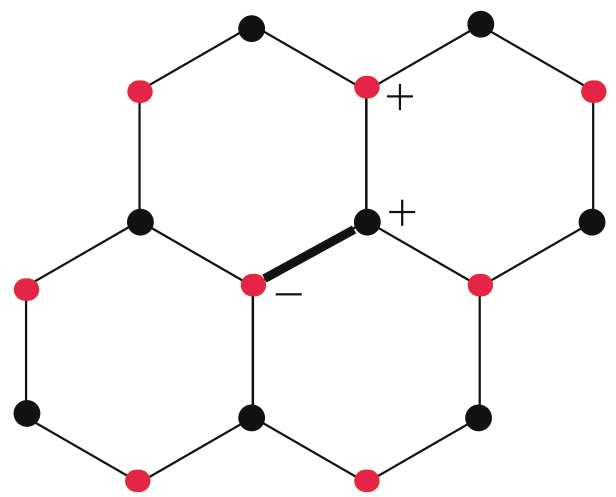

Fig. 4. Upon reversing the spins on one sublattice of the honeycomb lattice, $\tilde{K}_{p}$ and $\tilde{K}_{p^{\prime}}^{\prime}$ now penalize maximum and minimum $S^{z}$ eigenvalues respectively
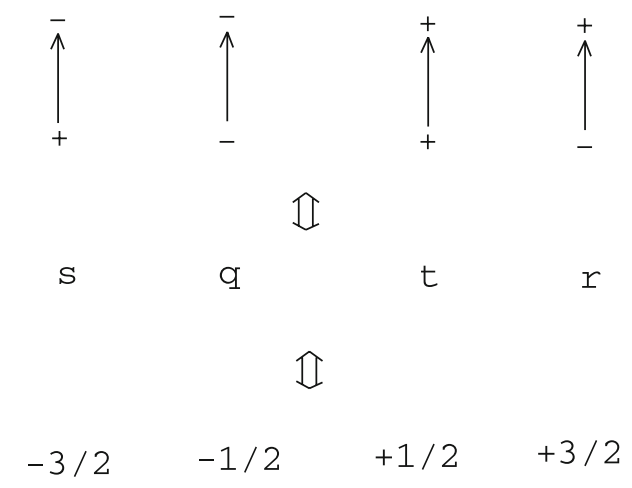

Fig. 5. The bottom row represents the spin eigenvalues

of $L^{\prime}$ ( $L$ and $L^{\prime}$ labeled as black and red respectively in Fig. 4)(in color on line only). $\tilde{K}$ is still necessarily 3-body but at least it now has the form of the Klein Hamiltonian, see e.g. [8]

$C$. The idea here is to take adjacent pairs of site spins from $B^{\prime}$ and encrypt them as the state of a spin $=3 / 2$ particle living on the bond $b$ joining the two sites. We orient $b$ from $L^{\prime}$ to $L$. Let us set up an indeterminate bijection (Fig. 5). We now "simulate" $\tilde{K}$ on a 


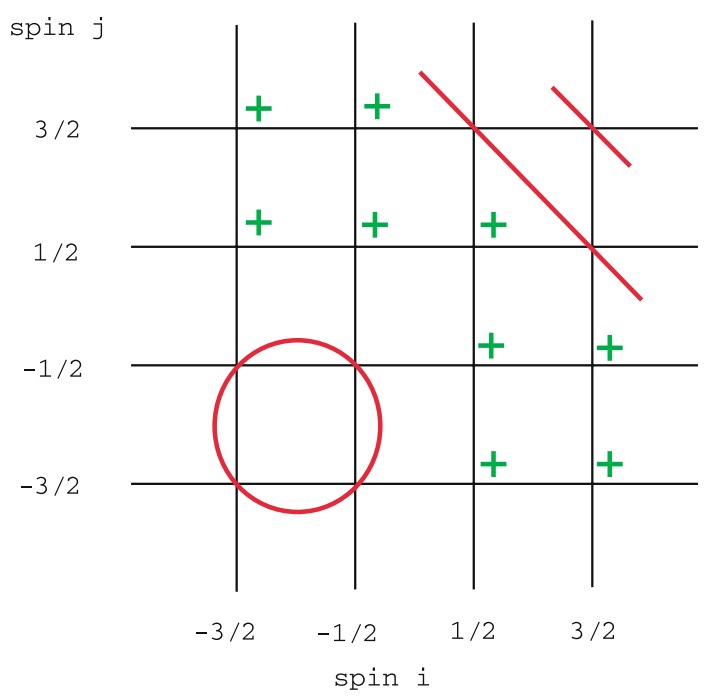

Fig. 6. The horizontal axis labels the $i^{\text {th }}$ spin and the vertical axis the $j^{\text {th }}$ spin

Hilbert space $\overline{\mathcal{H}}=\otimes_{\text {bonds }} C^{4}$, the space of a spin $=3 / 2$ particle on each bond. On sites $l \in L\left(l^{\prime} \in L^{\prime}\right)$ we must penalize $t \otimes t(q \otimes q)$.

Furthermore we must penalize inconsistent encryptions. For $l \in L\left(l^{\prime} \in L^{\prime}\right)$ the following pairs yield inconsistent site labels: $q \otimes r, r \otimes q, q \otimes t, t \otimes q, s \otimes r, r \otimes s, s \otimes t$, and $t \otimes s(q \otimes s, s \otimes q, q \otimes t, t \otimes q, r \otimes s, s \otimes r, r \otimes t$, and $t \otimes r)$.

We again obtain the space of nets as zero modes $\mathcal{H} \subset \overline{\mathcal{H}}$ by fixing $K$ to be the following 2-body Hamiltonian:

$$
\begin{aligned}
K= & \sum_{l \in L}\left(\Pi_{t \otimes t}+\Pi_{q \otimes r}+\Pi_{r \otimes q}+\Pi_{q \otimes t}+\Pi_{t \otimes q}+\Pi_{s \otimes r}+\Pi_{r \otimes s}+\Pi_{s \otimes t}+\Pi_{t \otimes s}\right) \\
& +\sum_{l^{\prime} \in L^{\prime}}\left(\Pi_{q \otimes q}+\Pi_{q \otimes s}+\Pi_{s \otimes q}+\Pi_{q \otimes t}+\Pi_{t \otimes q}+\Pi_{r \otimes s}+\Pi_{s \otimes r}+\Pi_{r \otimes t}+\Pi_{t \otimes r}\right),
\end{aligned}
$$

where $\Pi_{x \otimes y}$ denotes the projector onto the state $x \otimes y$.

We can write a Hamiltonian which effectively accomplishes such a projection in terms of the spins $S_{i}^{z}$ ( $i$ is the bond index). For black sites $H$ has terms

$$
\begin{array}{r}
\left(\left(S_{i}^{z}+1\right)^{2}+\left(S_{j}^{z}+1\right)^{2}-1 / 2\right) \\
\left(S_{i}^{z}+S_{j}^{z}-2\right)\left(S_{i}^{z}+S_{z j}-3\right)
\end{array}
$$

and for red sites

$$
\begin{gathered}
\left(\left(S_{i}^{z}-1\right)^{2}+\left(S_{j}^{z}-1\right)^{2}-1 / 2\right) \\
\left(S_{i}^{z}+S_{j}^{z}+2\right)\left(S_{i}^{z}+S_{j}^{z}+3\right) .
\end{gathered}
$$

The origin of these terms is illustrated in Fig. 6. 


\section{3. $\mathcal{H}, H$, and $V$}

From now on, we will be concerned with the properties of the Hilbert spaces $\mathcal{H}(\Sigma)$ on a surface $\Sigma$. If $\Sigma$ has boundary, $\partial \Sigma$, we fix a boundary condition by specifying points where the nets must end. In the case where $\Sigma$ has connected boundary and the boundary condition consists of $n$ points, we denote the Hilbert space by $\mathcal{H}(\Sigma, n)$. We will be interested in "isotopy invariant" wave functions $\Psi$, whose value is independent of deformation. (In Sect. 6 we relax this condition to allow certain bond fugacities.) To avoid unnormalizable wave functions, these loops should really live on a lattice, as in the previous section.

To produce invariant $\Psi$ 's we consider Hamiltonians $H: \mathcal{H} \rightarrow \mathcal{H}$ which contain fluctuations sufficient to enforce isotopy invariance (Fig. 7) on all low energy states. It should be remarked that it is not easy to set up such terms on a lattice; some fine tuning may be required (see [1] and [2] for a realization). Also there are questions of ergodicity - $H$ must have sufficient fluctuations that crystals do not compete with the liquid condition described by Fig. 7. Nevertheless we start by assuming these problems solved: that we have $\mathcal{H}$ and a family $\{H\}$ whose ground states $V$ consist of isotopy invariant wave functions $\Psi$.

The "axioms" we impose on the Hamiltonian $H$ are implicit in the following conditions that we require its ground state manifolds $V(\Sigma, n) \subset \mathcal{H}(\Sigma, n)$ to satisfy.

Axiom 1. $H$ is gapped-this makes $V(\Sigma, n)$ sharply defined.

Axiom 2. The following "minimal" dimensions on the 2-disk $\Sigma=D$ occur:

(i) $\operatorname{dim} V(D, 0)=1$,

(ii) $\operatorname{dim} V(D, 1)=0$,

(iii) $\operatorname{dim} V(D, 2)=1$,

(iv) $\operatorname{dim} V(D, 3)=1$,

(v) $\operatorname{dim} V(D, 4)$ takes the minimal value consistent with (i)-(iv) (to be computed below).

We make the further technical assumption that the constants $a, b$ and $c$ in Fig. 8 are neither 0 nor infinity.

Axiom 2(ii) is the "no tadpole" axiom which says that although Fig. $1 \mathrm{~b}$ ) is in $\mathcal{H}$ it has high energy. There is no low energy manifold $V$ whatsoever when the boundary condition only allows tadpoles. If one thinks in terms of $1+1$ dimensional physics, 2(ii) merely says the obvious: a single particle should not come out of the vacuum. If we nevertheless persisted in making $\operatorname{dim}(V(D, 1))=1$ we would admit the very boring

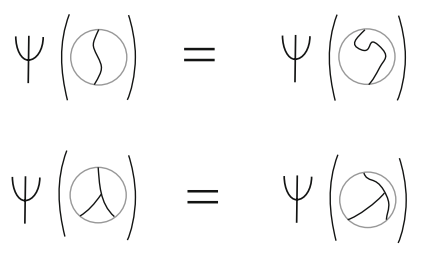

Fig. 7. The condition of isotopy invariance. Wavefunctions assign a complex amplitude for any string net (which is the amplitude for this configuration to occur). These equations mean that wavefunctions in the low-energy Hilbert space assign the same amplitude to two string nets if one can be obtained from the other by smooth deformations 


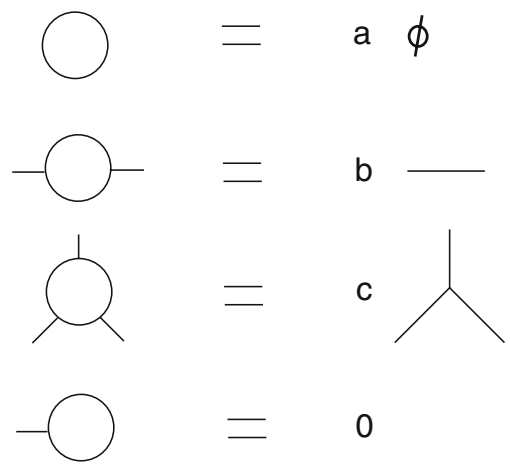

Fig. 8. We have stopped drawing the disk, but all diagrams above are nets in a 2-disk $D$ with the endpoints on D. Also we abuse notation to allow a net to also represent the ground state evaluation, $\Psi_{\mathrm{g} . \mathrm{s} .}$ (net)

case in which for all $\Sigma, \operatorname{dim}(V(\Sigma))=1$ and $V$ is spanned by the constant function on nets.

Similarly 2(i) and 2(iii) are required in a $1+1$ dimensional (unitary tensor category) context. Also, if either dimension is 0 then all $V$ have dimension 0 , by gluing formulae, so the entire theory collapses.

Axiom 2(iv) does represent a choice. If we instead said $\operatorname{dim}(V(D, 3))=0$, we would forbid our nets to branch. Here we know $Z_{2}$ gauge theory (i.e. the toric code) and the doubled semion theory — both abelian — can arise. Possibly higher doubled $S U(2)_{k}$ Chern-Simons theories might also arise from loop models, but entropy arguments [14] show that their ground state wave functions cannot be a simple Gibbs factor per loop [14]. So 2(iii) is not inevitable but represents our decision to set up whatever microscopics are necessary to build $(\mathcal{H}, H)$ with branched nets occurring in $V$, i.e. to build a string net model. We now derive:

Theorem 1. There is a unique theory (minimal category) $V(\Sigma)$ compatible with 1 and 2 above. It satisfies $\operatorname{dim}(V(D, 4))=2$ and is DFib, the doubled fibonacci category.

Proof. By "Axiom 2" there are nonzero constants $a, b, c \in \mathbf{C}$ such that the conditions in Fig. 8 hold.

Now consider the 4-point space $V(D, 4)$. If its dimension is $<4$ there must be relations among the 4 nets in the expression below (Fig. 9 a)), each thought of as an evaluation of the functional $\Psi_{\text {g.s. }}$ on $V(D, 4)$. We find conditions on the coefficients by joining various outputs of $\mathrm{R}$. This amounts to calculating consequent relations in the 1-dimensional spaces $V(D, 2)$ and $V(D, 3)$ which are implied by $R$. We work out, in part b) of Fig. 9 the implication of joining the upper outputs of $R$ by an arc. By such arguments, we also have the three relations in fig. (10). Eliminating $x$ and $y, x=-c h-b i, y=-b h-c i$, we obtain

$$
\begin{aligned}
(b-c-a b) h+(-b-a c) i & =0, \\
(-b-a c) i+(b-c-a b) h & =0 .
\end{aligned}
$$

Possible relations which can be imposed on string nets through the Hamiltonian correspond to solutions of this linear system. There can be at most two linearly independent solutions, and this case occurs exactly when the coefficients vanish: $b=c+a b, b=-a c$, 
(a)

$$
(\mathrm{R}=\mathrm{h})-(+\mathrm{iI}+\mathrm{x})(+\mathrm{y}
$$

(b)

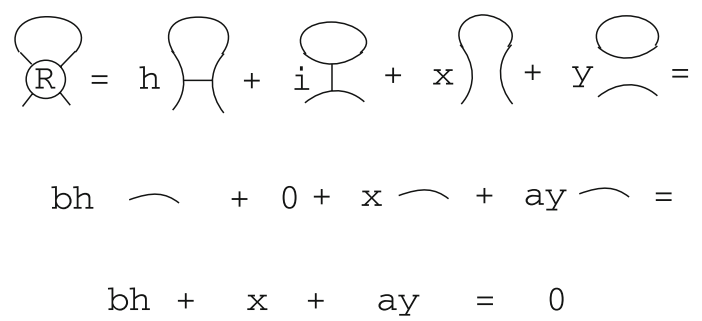

Fig. 9. If $V(D, 4)<4$, then there must be some coefficients $h, i, x, y$ such that the linear combination on the right-hand-side of (a) vanishes. By embedding these pictures within larger ones in such a way that the endpoints are connected as shown in (b) and using Fig. 8, we find relations satisfied by $h, i, x, y$

$$
\begin{aligned}
& \text { R }=0+i b+a x+y=0 \\
& \text { R }=0 \text { bh }+i b+x=0 \\
& R=0=>b h+c i+y=0
\end{aligned}
$$

Fig. 10. Three other relations obtained by connecting the endpoints of $R$

(a)

$$
)\left(=a^{-1} \rightleftharpoons+b^{-1} \Psi\right.
$$

(b)

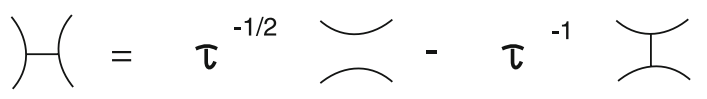

Fig. 11. The $6 j$ symbols can be obtained from the $h=0, i=1$ and $h=1, i=a^{-1}$ relations

so $-a c=c+\left(a^{2}\right) c$ or $a^{2}=a+1$. We already see the golden ratio $\tau$ emerge: $a=\tau$ or $-\tau^{-1}, \tau=\frac{1+\sqrt{5}}{2}$.

It is well known that all relations among ground state string net amplitudes are determined by $d$-isotopy and local reconnection rules encoded in the $6 j$ symbol or $F$-matrix (Fig. 11) (see e.g. [2]). In order to calculate the $6 j$-symbol, we set $h=0, i=1$ in $R$ to get the equation in Fig. 11 a). Unitarity requires $\left|a^{-1}\right|^{2}+\left|b^{-1}\right|^{2}=1$ so $a=\tau$ and $b=e^{2 \pi i \phi} \sqrt{\tau}$, where $\phi$ is an irrelevant phase (associated with identifying the simplest 3 -point diagram with some unit vector in $V(D, 3))$ which we set to 1 . 
Similarly setting $h=1, i=a^{-1}$ (and hence $x=0$ ) we find the second row of the $6 j$-symbol (i.e. the $F$ matrix) in Fig. 11 b) and thus:

$$
F=\left|\begin{array}{cc}
\tau^{-1} & \tau^{-1 / 2} \\
\tau^{-1 / 2} & -\tau^{-1}
\end{array}\right| .
$$

Thus, fixing $V(D, 4)$ to have minimal dimension, 2, we find our constants specified: $a=\tau, b=\tau^{1 / 2}, c=-\tau^{-1 / 2}$, and the $F$-matrix as well.

What we have obtained is the Turaev-Viro (or "doubled") version of the unitary Fibonacci fusion category. From these rules $-a, b, c$, and $F-$ all nets $G$ on a sphere can be evaluated to a scalar $\langle G\rangle_{\tau}$ which is the "golden" quantum invariant. Similarly, with this data the entire unitary modular functor is specified on all surfaces with or without boundary: $V \cong \mathrm{DFib}$.

Remark. If $V(D, 4)$ is allowed to have dimension 3, a generic solution, the Yamada polynomial, exists. It can be truncated to doubled $S O(3)_{k}$ - modular functors for $k$ odd $>3$ by imposing the correct dimension restriction on $V(D, 2 k)$. If $V(D, 4)$ is allowed to be 4-dimensional, a relation in $V(D, 5)$ realizes Kuperberg's $G_{2}$-spider which presumably admits further specializing relations which generate $G_{2}$ level $k$ topological quantum field theories (TQFTs).

\section{Deriving the Properties of DFib from $V(\Sigma, n)$}

In the remaining three sections of this paper, we will discuss the properties of the ground states $V(\Sigma, n) \subset \mathcal{H}(\Sigma, n)$, thereby obtaining physical properties of the topological phase DFib. DFib is the product of two copies of opposite chirality of the Fibonacci theory, Fib, which the simplest $2+1$ dimensional TQFT theory with nonabelian braiding rules. (Fib is also the simplest universal theory [13].) It arises as the "even sub-theory" of $S U(2)_{3}$ (i.e. integer spins only) or $S U(3)_{2}$ and also directly from $G(2)_{1}$.

Fib has one non-trivial particle $\tau$ with fusion rule:

$$
\tau \otimes \tau=1 \oplus \tau
$$

Here we use the term particle to refer to a representation of the corresponding affine Lie algebra $\left(S U(2)_{3}\right.$ for the Fibonacci theory). This is because such representations can be associated with Wilson lines in the Chern-Simons picture. Particles arise in the usual way, when the three dimensional space under consideration is the product of a 2 dimensional Riemann surface $\Sigma$ (thought of as space) and the real line (thought of as time). Specializing to Wilson lines tracing out stationary trajectories, we obtain the particles discussed here, and the degenerate ground states $V(\Sigma, n)$. Of course the quantum dimension of $\tau$ is $\frac{1+\sqrt{5}}{2}=\tau$. (In a slight abuse of notation, we use $\tau$ to denote both the particle and its quantum dimension, the golden ratio.)

A discrete manifestation of this quantum dimension is that $\operatorname{Fib}\left(S^{2}, n+2\right)$, the Hilbert space for $n+2 \tau$-particles at fixed position on the 2-sphere, is $\operatorname{Fib}(n)$, the $n^{\text {th }}$ Fibonacci number. (Proof. Fuse two of the particles: the result will be either $n+1$ or $n \tau$ 's on $S^{2}$ depending on the fusion process outcome. This yields the famous recursion formula: $\operatorname{dim} \operatorname{Fib}\left(S^{2}, n+2\right)=\operatorname{dim} \operatorname{Fib}\left(S^{2}, n+1\right)+\operatorname{dim} \operatorname{Fib}\left(S^{2}, n\right)$ which defines Fibonacci numbers.) This gives the exponential growth referred to in the introduction. 
Fib is a chiral theory with the following parameters:

$$
\begin{aligned}
S & =\frac{1}{\sqrt{\tau+2}}\left|\begin{array}{cc}
1 & \tau \\
\tau & -1
\end{array}\right|, \\
S_{\tau \tau}^{\tau} & =e^{3 \pi i / 10}, \\
F & =\left|\begin{array}{cc}
\tau^{-1} & \tau^{-1 / 2} \\
\tau^{-1 / 2}-\tau^{-1}
\end{array}\right|, \\
& =\exp (4 \mathrm{pi} \mathrm{i} 5)
\end{aligned}
$$

The theory $V$ constructed in Sect. 3 is isomorphic to Fib* $\otimes$ Fib $\cong$ End(Fib). Very briefly we explain this connection in the context of a closed surface $\Sigma$ (visualize $\Sigma=$ torus). Let $G$ be a fine net on $\Sigma$ which "fills it" in the sense that all complementary regions are disks $\left\{\delta_{i}\right\}$. According to Reshetikin-Turaev [16], labelings (by 1 or $\tau$ ) of the bonds of $G$ consistent with Fib fusion rules span the Hilbert space for a very high genus surface $\Sigma^{\dagger}$ which is the boundary of $\bar{G},(\bar{G}$ is a 3-D thickening of the net $G)$. It can be seen that the fixed space under the $F$-matrix action on $G$-labelings can be obtained from projectors associated to $\left\{\delta_{i}\right\}$. These disks determine projectors, or plaquette operators, onto the trivial particle type along a collection of "longitudes" on $\Sigma^{\dagger}$ (where $\delta_{i}$ intersects $\Sigma^{\dagger}$ ). Another way to implement these plaquette operators is to add $\left\{\bar{\delta}_{i}\right\}$ (thickenings of $\left\{\delta_{i}\right\}$ to $\bar{G}$ ). $\bar{G} \bigcup\left\{\bar{\delta}_{i}\right\}$ is homeomorphic to a product $\Sigma \times I$, surface cross interval. Adding the plaquet operators has cut $\operatorname{Fib}\left(\Sigma^{\dagger}\right)$ down to $\operatorname{Fib}(\partial(\Sigma \times I))=$ $\operatorname{Fib}(\bar{\Sigma} \bigsqcup \Sigma)=\operatorname{Fib}^{*}(\Sigma) \otimes \operatorname{Fib}(\Sigma)=\operatorname{DFib}(\Sigma)$.

From this point of view the double arises from the fact that surface $\times I$ has both an "inner" and "outer" boundary. It is nontrivial to align the various structures (e.g. particles) of DFib from the two perspectives: one as a theory of trivalent graphs on a surface (Sect. 3) and the other as a tensor product of a chiral theory and its dual. For this reason we are not content to merely state that the particle content of DFib is $1 \otimes 1,1 \otimes \tau, \tau \otimes 1$, and $\tau \otimes \tau$. Rather, we will give a direct string representation for these particles shortly.

Remark. The expression of DFib (and more generally, th Turaev-Viro theories) through commuting local projectors was known to Kitaev and Kuperberg and made explicit for DFib in [1]. A conceptual understanding of the commutation relations is readily at hand from the preceding construction of $\Sigma \times I$. The "longitudes" on which we apply plaquette projectors are disjoint and thus commuting. The fusion rules that are enforced at disjoint vertices also commute. Finally vertex and plaquet terms commute because rules are preserved under the addition of an additional ("passive") particle trajectory, labeled $d$ in Fig. 12.

We conclude this section by finding the 4 irreducible representations of the DFib annulus category. This is the linear $C^{*}$-category whose objects are finite point sets 

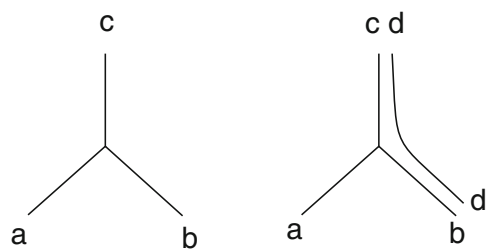

Fig. 12. The addition of a passive arc to the (piece of a) string net on the right preserves the rules satisfied by nets

(a)

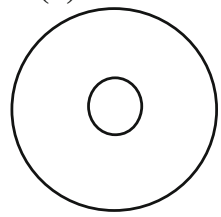

(b)

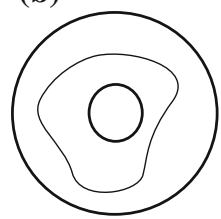

Fig. 13. a) $1 \in A_{0,0}$. b) $R \in A_{0,0}$

(boundary data) on a circle and whose morphisms are formal combinations of nets in the annulus, which obey the linear rules $a, b, c$, and $F$, and which mediate between the boundary data.

The four irreducible category (or "Algebroid") representations are detected as idempotents in $A_{0,0}$ and $A_{1,1}$, the algebra under stacking of nets in annuli with either trivial or 1-point boundary data. A table which organizes the results and compares back to the Fib $^{*} \otimes$ Fib picture is given below. The entries show the dimensions of the Hilbert space of (formal combinations of) nets on an annulus which start on the inner boundary with a given boundary condition (horizontal axis) and terminate near the outer boundary with a copy of a given idempotent (vertical axis).

\begin{tabular}{lllll} 
& \multicolumn{3}{l}{ boundary conditions } & \\
irreps (idempotents) & 0 & 1 & 2 & $\ldots$ \\
\hline$e_{1} \cong 1 \otimes 1$ & 1 & 0 & $>0$ & $>0$ \\
$e_{2} \cong \tau \otimes \tau$ & 1 & 1 & $>0$ & \\
$e_{3} \cong 1 \otimes \tau$ & 0 & 1 & $>0$ & \\
$e_{4} \cong \tau \otimes 1$ & 0 & 1 & $>0$
\end{tabular}

Let us start by finding the idempotents for $A_{0,0} \cdot A_{0,0}$ has the empty net as its identity and is generated by a single ring $R$. The $a, b, c, F$ rules show: $R^{2}=1+R$ so $A_{0,0} \cong C[R] /\left(R^{2}=1+R\right)$.

More generally, the idempotents in the algebra $C[x] / P(x), P(x)=\left(x-a_{1}\right) \cdots(x-$ $a_{k}$ ), all roots distinct, are given by:

$$
e_{i}=\frac{\left(x-a_{i}\right) \cdots\left(\widehat{x-a_{i}}\right) \cdots\left(x-a_{k}\right)}{\left(a_{i}-a_{1}\right) \cdots\left(\widehat{a_{i}-a_{i}}\right) \cdots\left(a_{i}-a_{k}\right)} .
$$

We get

$$
\begin{aligned}
& e_{1}=\frac{1+\tau R}{\tau+2}(\cong 1 \otimes 1, \text { the trivial particle }), \\
& e_{2}=\frac{1+\bar{\tau} R}{2+\bar{\tau}}(\cong \tau \otimes \tau),
\end{aligned}
$$


(a)

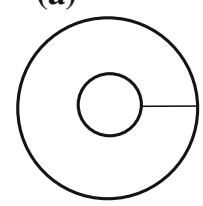

(b)

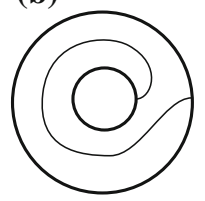

(c)

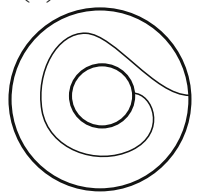

Fig. 14. a) $1 \in A_{1,1}$, b) $T \in A_{1,1}$, c) $T^{-1} \in A_{1,1}$

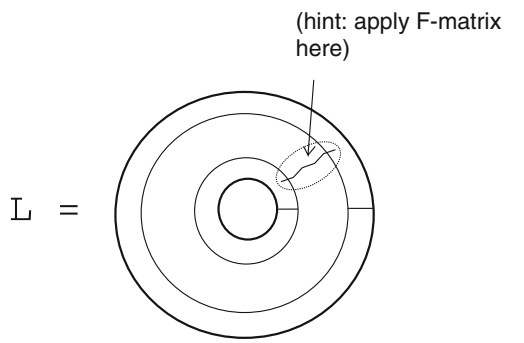

Fig. 15. A compact representation of $L=\tau^{-1 / 2} 1+\tau^{-3 / 2}\left(T+T^{-1}\right)$, as may be seen by applying the $F$-matrix where indicated
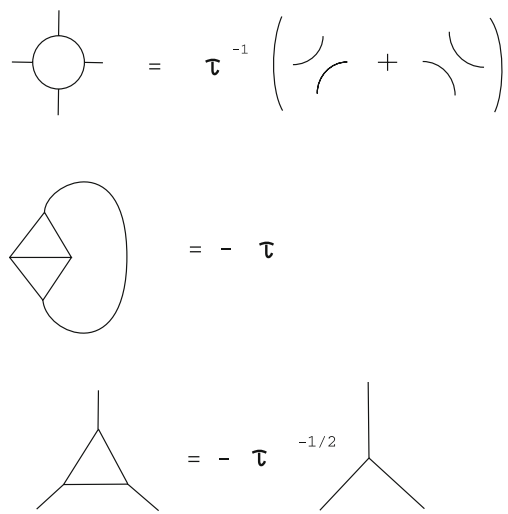

Fig. 16. The relations depicted above can be obtained by applying the $a, b, c$, and $F$ rules

$\bar{\tau}=-\tau^{-1}$. Using the $a, b, c$, and $F$ rules the algebra $A_{1,1}$ is seen to be generated by the identity $1, T$, and $T^{-1}$ (where $T$ is defined in Fig. 14). There is an element $L=\tau^{-1 / 2} 1+\tau^{-3 / 2}\left(T+T^{-1}\right)$ which factors in a category sense through $A_{0,0}$. In fact, using the elementary rules in Fig. 8 we derive the useful identities in Fig. 16 and we find that $L$ is equivalently represented as shown in Fig. 15. It follows that to find a new representation of the annulus category we should look for the idempotents in $A_{1,1} / L$. $A_{1,1} / L \cong C[T] /\left\{T^{2}+\tau T+1=0\right\}$. So, using (11) again and a little manipulation we find idempotents

$$
\tilde{e}_{3}=\frac{T-\left(\frac{\tau-\sqrt{\tau-3}}{2}\right) 1}{(-\sqrt{\tau-3})}
$$


and

$$
\tilde{e}_{4}=\frac{T+\left(\frac{\tau+\sqrt{\tau-3}}{2}\right) 1}{\sqrt{\tau-3}}
$$

in the quotient algebra. Also $L^{2}=\left(\tau^{1 / 2}+\tau-3 / 2\right) L$, so $e_{L}=L / \tau^{1 / 2}+\tau^{-3 / 2}$, so again using the identities in Fig. 16 we can find $e_{3}=\tilde{e}_{3}\left(1-e_{L}\right)$ and $e_{4}=\tilde{e}_{4}\left(1-e_{L}\right)$ :

$$
\begin{aligned}
& e_{3}=\tilde{e}_{3}+\frac{\tau+2+\sqrt{\tau-3}}{2\left(\tau^{1 / 2}+\tau^{-3 / 2}\right) \sqrt{\tau-3}} L, \\
& e_{4}=\tilde{e}_{4}+\frac{-(\tau+2)-\sqrt{\tau-3}}{2\left(\tau^{1 / 2}+\tau^{-3 / 2}\right) \sqrt{\tau-3}} L .
\end{aligned}
$$

\section{Chromatic Polynomial and Yamada Polynomial}

The relation between the chromatic polynomial and ground state amplitudes of string nets has been studied before in [2]. In this section we use an identity for the chromatic polynomial (Tutte's "golden ration theorem") to deduce a simpler looking version of such a relation. The chromatic polynomial $\chi_{\hat{G}}(k)$ of a graph $\hat{G}$ at the positive integer $k$ counts the number of $k$-colorings of the vertices of the graph (so that no two vertices connected by a bond are given the same color). $\chi$ obeys the famous "delete-contract" recursion relation:

$$
\chi_{\hat{G}}(k)=\chi_{\hat{G}-e}(k)-\chi_{\hat{G} / e}(k) .
$$

This relation can be depicted graphically as shown in Fig. (17), in which we have suppressed $\chi$ (as we have consistently suppressed the wave function and written the relation out in terms of its pictorial argument). This is a local relation; there is no significance to the bit of $\hat{G}$ near the active edge $e$, which in the drawing is represented, purely for illustrative purposes, by three half-edges on top and bottom. $\chi(k)$ is completely fixed by (18) and the following conditions: that $\chi$ vanish on any graph in which the two ends of

(a)

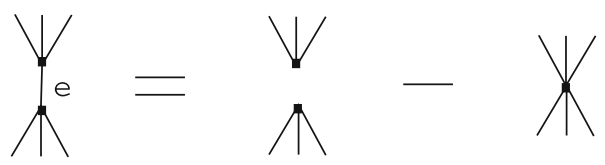

(b)

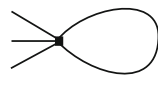

(c)

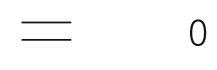

0

$\mathrm{k}$

Fig. 17. (a) Graphical depiction of the "delete-contract" recursion relation. This relation together with the two depicted graphically in (b) and (c) and multiplicativity under disjoint union completely determine $\chi_{\hat{G}}{ }^{(k)}$ 


$$
2\left|=\frac{\mid 1}{\frac{P_{2}}{\mid T}}=\right|-1 / \mathrm{d} \bigcap
$$

Fig. 18. Jones-Wenzl projector

$$
\begin{aligned}
& \langle\theta \|\rangle_{d}=\frac{2}{2}= \\
& =a^{3}-3 d a+3 / a^{2} S 5-1 / d^{3} 55 \\
& =a^{3}-3 a+2 a^{-1}-a^{-1}
\end{aligned}
$$

Fig. 19. The Yamada polynomial for the $\theta$ graph

a single bond are joined to the same vertex, that $\chi_{\text {single pt. }}(k)=k$, and multiplicativity under disjoint union:

$$
\chi_{\hat{G} \amalg \hat{H}}=\chi_{\hat{G}} \chi_{\hat{H}} \cdot
$$

We will be interested in $\chi_{\hat{G}}$ evaluated at noninteger values as well as integral ones.

We now turn to the Yamada polynomial defined for a net (trivalent graph) $G$ lying in the plane (or 2-sphere); we denote it by $\langle\langle G\rangle\rangle_{d}$, where $d$ is the variable. To define $\langle\langle G\rangle\rangle_{d}$, recall the 2-strand Jones-Wenzl projector, an idempotent familiar from the study of the Temperley-Lieb algebra $T L_{d}$. Given $G,\langle\langle G\rangle\rangle_{d}$ is defined by labeling every arc of $G$ by 2 as in (18) and then expanding to a weighted superposition of multi-loops. In each term, each loop contributes a numerical factor of $d$,

$$
\left.\langle\langle G\rangle\rangle_{d}=\sum_{\text {terms }} \text { (coeff. }\right) d^{\# \text { loops }} .
$$

For example, if $G$ is a graph shaped like the Greek letter $\theta$, we have the result shown in Fig. 19. As this example shows, the Yamada polynomial is actually a polynomial in $d, d^{-1}$.

Theorem 2. If $G$ is a net in the 2-sphere and $\hat{G}$ is the dual graph then:

$$
\langle\langle G\rangle\rangle_{d}=d^{-V(\hat{G})} \chi_{\hat{G}}\left(d^{2}\right),
$$

$V=$ number of vertices of $\hat{G}=$ number of faces of $G$.

Proof. The above procedure for turning a net into a superposition of multi-loops may be generalized by declaring two local rules shown in Fig. 20. The first rule says a point is replaced by a circle with a possible numerical weight (vertex fugacity) and each arc 


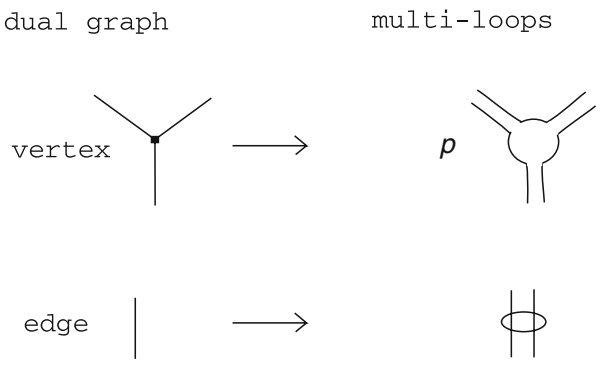

Fig. 20. Local rules for turning the dual graph of a net into a superposition of multi-loops. The ellipse represents an unknown combination

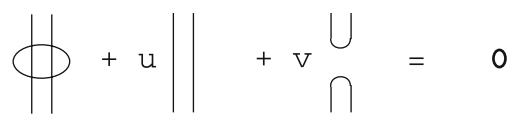

Fig. 21. A recoupling rule by which the dual of a trivalent graph can be converted into multi-loops. We will choose $u$ and $v$ (and also $\rho$ ) so that the procedure for making a trivalent graph into a multi-loop relates $\chi_{\hat{G}}(k)$ and $\langle\langle G\rangle\rangle_{d}$
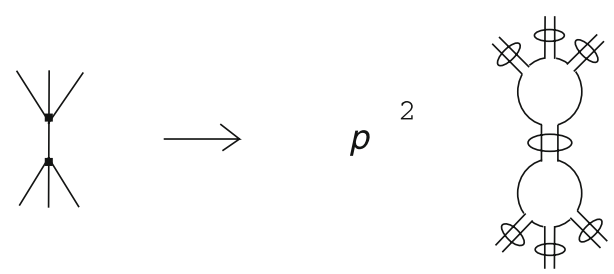

Fig. 22. Applying the rules in Fig. 20 to the graph on the left-hand side of the figure above yields the picture on the right

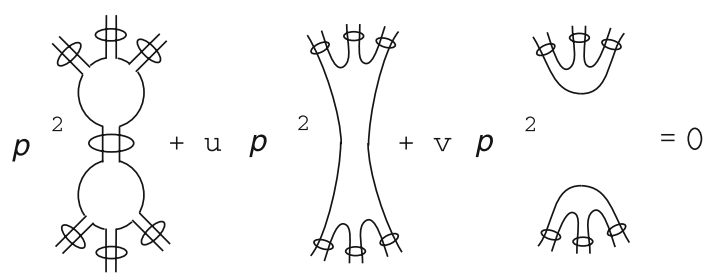

Fig. 23. Using the recoupling rule in Fig. 21, we can simplify the picture on the right-hand-side of Fig. 22

is replaced by two lines with some general recoupling. We express the fact that this recoupling is still a variable to be solved for by Fig. 21. Our goal is to find suitable values for $\rho, u$, and $v$ so that $\chi_{\hat{G}}(k)$ comes out related to $\langle\langle G\rangle\rangle_{d}$. The correct $k$ will turn out to be $d^{2}$.

Let us look near a typical edge $e$ of $\hat{G}$ and expand it using the rule in Fig. 22.

With this expansion and Fig. 21 we obtain Fig. 23. Translating this back into graphs yields Fig. 24. Also, since graphs with an edge that connects a vertex to itself evaluate to zero, we have Fig. 25, implying $v=-u d$. Also, $k=\rho d$.

Comparing Fig. 24 with the chromatic relation (18) we find: $v=-1, u=1 / \rho$. Because we have $v=-u d$, we obtain $u=1 / d$ so $d=\rho$. Finally, since $k=\rho d$, $k=d^{2}$. The mystery combination turns out to be a "sideways" $P_{2}$ as shown in Fig. 26. 


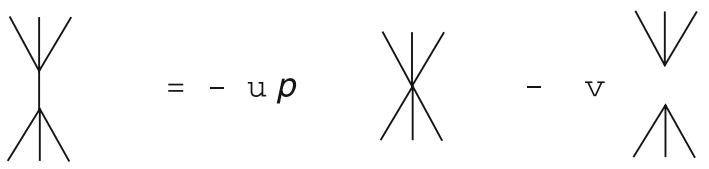

Fig. 24. A relation that must be satisfied by $u, v$, and $\rho$

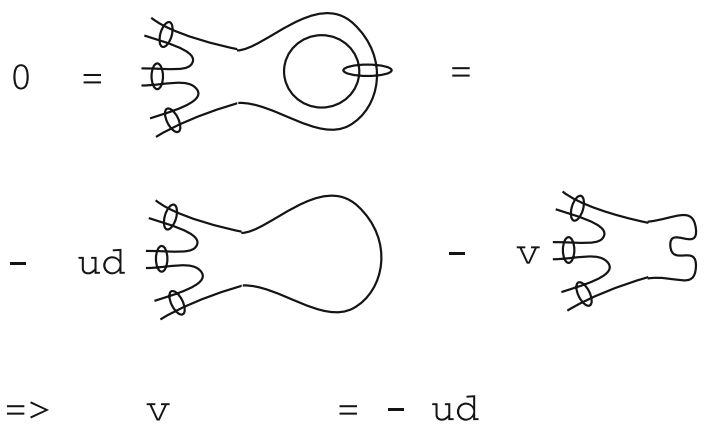

Fig. 25. A graph with an edge that connects a vertex to itself evaluates to zero, from which we deduce a relation between $u$ and $v$

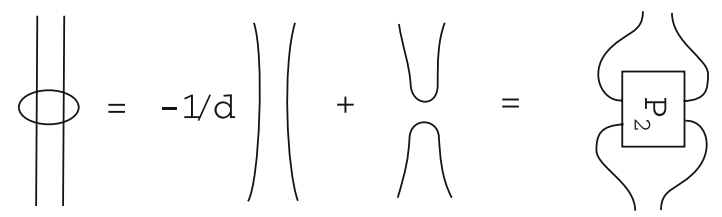

Fig. 26. Solving for $u, v, \rho$, we see that the recoupling rule is just a "sideways" $P_{2}$

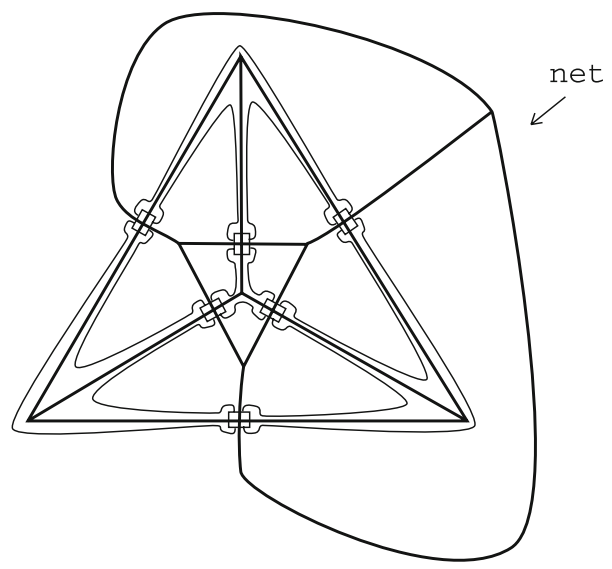

Fig. 27. Applying the rules to a complete graph

To complete the proof, it remains to see the global geometry of how these $P_{2}$ 's hook together. We claim that they lie along (doubled) dual graph edges. It suffices to examine an example. We take $\hat{G}$ and $G$ to be the complete graph $C_{4}$ shown in Fig. 27. The 
factor of $\rho^{V(\hat{G})}$ appearing with $\langle\langle G\rangle\rangle_{d}$ has been put on the right-hand-side in the theorem statement.

It is well known that the Yamada polynomial and the unitary invariant $\langle G\rangle_{\tau}$ of Sect. 3 are closely related. Specifically, when $d=\tau$, we should modify the Yamada polynomial $\langle\langle G\rangle\rangle_{\tau}$ by a vertex fugacity to obtain $\langle G\rangle_{\tau}$. This can be seen by checking that both quantities satisfy the elementary rules of Sect. 3 that determine them uniquely. From our example, shown in (19), we find $\langle\langle\theta\rangle\rangle_{\tau}=\tau^{-1}$ while for the unitary theory $\langle\theta\rangle_{\tau}=a b=\tau^{3 / 2}$. To convert from the unnormalized (Kauffman) theory to the unitary theory (compare to [1]) one must multiply in a factor of $\tau^{5 / 4}$ for each vertex. Thus:

$$
\langle G\rangle_{\tau}=\left(\tau^{5 / 4}\right)^{V(G)} \tau^{-V(\hat{G})} \chi_{\hat{G}}\left(\tau^{2}\right) .
$$

Using the Euler relation $F(\hat{G})+V(\hat{G})-E(\hat{G})$ and the fact that $\hat{G}$ is a triangulation, implying $E=\frac{3}{2} F$, we find:

$$
F(\hat{G})=2 V(\hat{G})-4
$$

Therefore:

$$
\langle G\rangle_{\tau}=\tau^{-5} \tau^{\frac{3}{2} V(\hat{G})} \chi_{\hat{G}}(\tau+1) .
$$

We can now use Tutte's "golden ratio theorem" ([T] and [L]) ([9]): For a planar triangulation $\hat{G}$ :

$$
\left(\chi_{\hat{G}}(\tau+1)\right)^{2} \tau^{3 V}(\tau+2)\left(\tau^{-10}\right)=\chi_{\hat{G}}(\tau+2) .
$$

This formula allowed Tutte to conclude that the r.h.s. is positive, creating a curious analogue (and precursor) to the 4-color theorem. (Neither result has been shown to imply the other.)

Now square (24) and substitute into (25); the result is a remarkable formula:

$$
\left(\langle G\rangle_{\tau}\right)^{2}=\frac{1}{\tau+2} \chi_{\hat{G}}(\tau+2)
$$

We have just proved the formula when $\hat{G}$ is a triangulation; in fact it holds more generally whenever $G$ is a net. To establish this it suffices to check the formula when $G$ is a single loop and to observe that the formula behaves well under disjoint union of disconnected components of $G$ : the l.h.s. is obviously multiplicative and, it turns out, so is the r.h.s. The reason is that disjoint union of $G_{1}$ and $G_{2}$ corresponds to a 1-point union $\hat{G}_{1} \bigvee \hat{G}_{2}$, and with the factor of $1 /(\tau+2)$, the chromatic polynomial becomes multiplicative under 1-point unions. So we have proved:

Theorem 3. Let $G$ be a net in the plane or 2-sphere (possibly disconnected and possibly with circle components). Then

$$
\langle G\rangle_{\tau}{ }^{2}=\left(\frac{1}{\tau+2}\right) \chi_{\hat{G}}(\tau+2) .
$$

Note: We would like to thank P. Fendley and E. Fradkin for a discussion of this identity. In their paper [2], a non-unitary normalization for $\langle G\rangle_{\tau}$ led to a vertex fugacity on the right-hand-side of (27), thereby obscuring the simplicity of (27) and the direct connection to the Potts model. 


\section{String Net Wavefunctions and the Potts Model}

Let us review the high- and low-temperature expansions of the Potts model. We begin by assuming a lattice with $Q$ "spin values" $\sigma_{i}=0,1, \ldots, Q-1$ at each site $i$. The partition function $Z(Q)$ is defined by:

$$
\begin{aligned}
Z & =\sum_{\sigma} \exp \left(-\beta\left(-J \sum_{\langle i, j\rangle} \delta_{\sigma_{i}, \sigma_{j}}\right)\right) \\
& =\sum_{\sigma} \prod_{\langle i, j\rangle} \exp \left(\beta J \delta_{\sigma_{i}, \sigma_{j}}\right),
\end{aligned}
$$

where the sum is over spin state configurations. Setting $\gamma=e^{\beta J}-1$ and expanding in powers of $\gamma$ we obtain the high-temperature expansion:

$$
Z=\sum Q^{c} \gamma^{b}
$$

where the sum is over bond configurations; $c$ is the number of clusters, and $b$ is the number of bonds. From now on, we consider the ferromagnetic case $J=1$. Note that the last sum is over the $2^{b}$ distinct subsets, not the $Q^{(\# \text { of sites })}$ distinct colorings because a Fubini resummation has taken place. Also, note that isolated sites count as clusters in (30). This is the Fortuin-Kateleyn representation [17]. It is known that for $0<Q \leq 4$ the model is critical precisely at its self-dual point, $\gamma=\sqrt{Q}$.

The high temperature expansion has been used [2,7] to study loop gases with ground state wavefunction whose amplitude is $d^{L}$ for some real number $d$, where $L$ is the number of loops. The square of such a wave function can be interpreted as a Gibbs weight $\left(d^{2}\right)^{L}$ providing a "plasma analogy" between topological ground states and the statistical physics of loop gases. We can easily see that a loop gas with Gibbs weight $d^{2}$ per loop is critical if $d \leq \sqrt{2}$ :

$$
\left(d^{2}\right)^{L}=\left(d^{2}\right)^{c+c^{*}}=\left(d^{2}\right)^{2 c+b}=\left(d^{4}\right)^{c}\left(d^{2}\right)^{b} .
$$

Here, $c^{*}$ is the number of dual clusters, i.e. the minimum number of occupied bonds which have to be cut in order to make each cluster tree-like (essentially the number of "voids" which are completely contained within clusters). The first equality follows from each loop being either the outer boundary of a cluster or a dual cluster. The second is due to the Euler relation $c^{*}=c+b+$ const. To map this squared wavefunction to the Potts model, we need $d^{4}=Q \leq 4$; note the edge fugacity for this loop model is automatically $\sqrt{Q}$, placing the model at its self dual point.

The low-temperature expansion of the Potts model is:

$$
Z(Q)=\sum_{G} \chi_{\hat{G}}(Q)\left(e^{-\beta J}\right)^{L},
$$

where the sum over graphs $G$ and $L$ is the total length of the graph. We specialize to the case in which the dual lattice is trivalent (e.g. the triangular lattice, whose dual lattice is the honeycomb), so that the graphs are all trivalent. At the critical point $(Q \leq 4)$, this becomes

$$
Z(Q)=\sum_{G} \chi_{\hat{G}}(Q)\left(\frac{1}{\sqrt{Q}+1}\right)^{L},
$$

using the condition for criticality $e^{\beta_{c} J}-1=\sqrt{Q}$. 
The (unnormalized) isotopy invariant wave function $\Psi$ constructed in Sect. 3 satisfies $\Psi(G)=\langle G\rangle_{\tau}$. Hence the corresponding statistical physics (of equal time correlators) is that of the normalized probability distribution:

$$
\operatorname{prob}(G)=\frac{1}{Z(Q=\tau+2, \beta=0)}\left(\langle G\rangle_{\tau}\right)^{2} .
$$

Note that (using (27)) this is the high temperature limit of the low temperature expansion.

This situation reminds us of the Toric code [18], where the weight on loops may be obtained by setting $Q=2, \beta=0$ (for $Q=2$, branched nets have zero weight), so the toric code ground state wavefunction can also be understood in terms of the hightemperature limit of the low-temperature expansion of the Potts model. In both cases, we conjecture that for $\beta<\beta_{c}$ the wavefunction with isotopy invariance modified by:

$$
\Psi(G)=\langle G\rangle_{\tau} x^{\text {length }(G)}
$$

for $1 \geq x>1 /(\sqrt{Q}+1)(\approx 0.345$ when $Q=\tau+2$, and $\approx 0.466$ when $Q=2)$ will be the ground state for some gapped Hamiltonian in the corresponding topological phase DFib (or Toric code).

Numerical work [19] already supports this conjecture in the Toric code case. Also, note that at $\beta>\beta_{c}$ (so $\left.x<1 /(\sqrt{Q}+1)\right)$ an effective string tension prevents the nets from fluctuating. Thus, the system leaves the topological phase and enters a "geodesic phase" in which small nets dominate. Recoupling is now unlikely, so the necessary topological relations are not well enforced and geodesic continuation allows states on the torus to be fairly well guessed by measurement within a subdisk violating the disk axiom. Finally, (27) identifies the ground state of the gapped Levin-Wen [1] Hamiltonian: $\Psi(G)=\langle G\rangle_{\tau}$ as the $\beta=0$ end point of the conjectured family.

In ref. [2], it is conjectured that the unnormalized probability distribution on nets $\left(\langle\langle G\rangle\rangle_{d}\right)^{2}$ should somehow yield the same statistical physics as the Potts model at an "effective" $Q_{\text {eff }}$ satisfying:

$$
\left(Q_{\text {eff }}-1\right)=\left(d^{2}-1\right)^{2} .
$$

If we set $d=\tau$ and adjust the $G$-vertex fugacity so as to replace $\langle\langle G\rangle\rangle_{\tau}$ with $\langle G\rangle_{\tau}$, as we did in Sect. 5, then the conjecture is precisely verified at $Q=\tau+2, \beta=0$ :

$$
(\tau+2-1)=\left(\tau^{2}-1\right)^{2} .
$$

We close this section with an observation whose importance, if any, we do not yet understand. The high temperature expansion weights loops while the low temperature expansion weights nets. But loops are nets, so we might ask: at what value of $Q$ do the high and low temperature expansions weigh loops equally? In the low temperature expansion, loops have weights $Q-1$ and in the high temperature expansion they have weight $\sqrt{Q}$ (from (31)). So loops are equally weighted when $Q-1=\sqrt{Q}$, i.e. when $Q=\tau^{2}$.

\section{Conclusions}

DFib is among the simplest conceivable achiral particle theories. In some sense it rivals the toric code in simplicity (both have 4 particles), but is vastly richer (in fact, universal [13]) in its braiding. We have explored the path to this phase, taking a Hilbert space $\mathcal{H}$ of nets and an isotopy invariant Hamiltonian (possibly with a bond fugacity, see Sect. 6) $H: \mathcal{H} \rightarrow \mathcal{H}$ as our starting point. The exploration has been combinatorial in Sect. 3, algebraic in Sect. 4, and statistical in Sect. 6. 
In Sect. 3, we saw that DFib emerges from minimizing certain degeneracies. This encourages us to believe the phase will ultimately be found in nature - as nature abhors a degeneracy.

In Sect. 6, we establish that the nets $G$ with (squared) topological weighting $\left(\langle G\rangle_{\tau}\right)^{2}$ (as usual, squaring the wavefunction to obtain a probability) are in a high temperature phase of the $(\tau+2)$-state Potts model (above criticality). This is also encouraging: the classical critical point looks as if it is the "plasma analogy" of a quantum critical point sitting at the entrance to the DFib phase. A parallel is explored between this situation and the $Q=2$ Potts critical point which serves as an entrance to the toric code phase.

Unresolved is what, more precisely, is required of $H: \mathcal{H} \rightarrow \mathcal{H}$ to be in the DFib phase. Is enforcement of the net $G$ structure (encoded in the definition of $\mathcal{H}$ ) plus strong dynamic fluctuation of $G$ adequate? We do not know.

Acknowledgement. We would like to thank Paul Fendley and Eduardo Fradkin for useful discussions. C.N. would like to acknowledge the support of the NSF under grant no. DMR-0411800 and the ARO under grant W911NF-04-1-0236 (C.N.). This research has been supported by the NSF under grants DMR-0130388 and DMR-0354772 (Z.W.). L.F. would like to acknowledge the support of the NSF under grant no. PHY -0244728.

Open Access This article is distributed under the terms of the Creative Commons Attribution Noncommercial License which permits any noncommercial use, distribution, and reproduction in any medium, provided the original author(s) and source are credited.

\section{Appendix A: The Chromatic Polynomial and Hard Hexagons}

In this appendix we extend a theorem of Tutte [9] which bounds the decay of the chromatic polynomial at $\tau+2$ of planar graphs. Specifically, we obtain a better sharp bound for graphs that consist of a large regions of hexagonal lattice (see Fig. 28). The ground state of the doubled Fibonacci theory DFib consists of a certain superposition of string net configurations on a hexagonal lattice. As in ref. [2] we may construct from it a classical statistical mechanical model of nets, with the Boltzmann weight of each net equal to the norm squared of its ground state amplitude. As discussed in Sect. 5, this statistical mechanical model is the (solvable) infinite-temperature limit of the $Q=\tau+2$ Potts model. We are interested in the rough quantitative behavior of the amplitudes for different types of graphs. In particular, we can consider a large chunk of hexagonal lattice, which is just the string net consisting of a large finite region whose bulk includes every available bond (see Fig. 28). We can ask about how its amplitude scales with the size of the region. Now, the Boltzmann weight of a string net is (up to overall scaling) just the chromatic polynomial at $\tau+2$ of the graph dual to the net, which can be interpreted

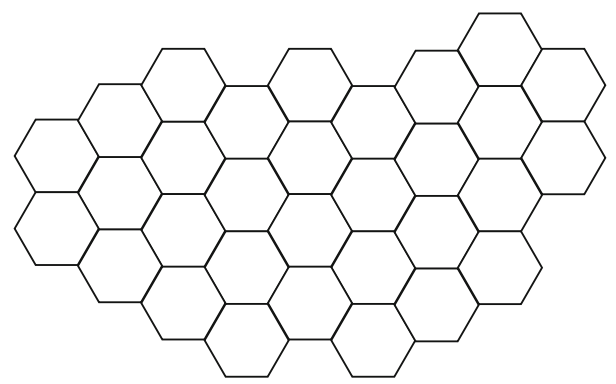

Fig. 28. Large chunk of hexagonal lattice 
as the zero-temperature limit of the $\tau+2$ antiferromagnetic Potts model defined on the graph dual to the net. So for the large chunk configuration, we are just interested in the behavior of the zero-temperature free energy of the $\tau+2$ antiferromagnetic Potts model on the hexagonal lattice.

This model is critical [11] and is expected to be described by a conformal field theory. On general grounds [10] we expect the free energy to scale like

$$
F=c_{0} A+c_{1} L+c_{2} \log L,
$$

where $c_{2}$ is universal and related to the central charge of the CFT and $c_{0}$ and $c_{1}$ are non-universal (here $A$ is the area, i.e. number of hexagons in the region, and $L$ is the length of the boundary). It turns out that the model is exactly solvable and we can obtain an exact analytic expression for $c_{0}$.

To do so, we first use the so-called shadow method [20] to evaluate $G_{\tau}$ of a net. This method works as follows. We take the net to be located on the sphere and assume that the regions (or faces) that it bounds are simply connected and do not border themselves. Then the shadow method (applied to DFib) gives $G_{\tau}$ as a sum over black and white colorings of the faces

$$
G_{\tau}=\sum_{\text {colorings C }} F_{C} E_{C}^{-1} V_{C} .
$$

The colors black and white are identified with the two particle types of the theory, and

$$
\begin{aligned}
F_{C} & =\prod_{\text {faces } \mathrm{F}} d_{C(F)}, \\
E_{C} & =\prod_{\text {edges } \mathrm{E}} \Theta(C, E), \\
V_{C} & =\prod_{\text {vertices } \mathrm{V}} \text { Tetrahedron }(C, V) .
\end{aligned}
$$

Here $d_{C(F)}$ stands for the quantum dimension associated with the color of face $F$ (1 for black, $\tau$ for white). $\Theta(C, E)$ is the theta graph along whose three edges run particle types associated with the edge $E$ (always the nontrivial particle) and the two faces which $E$ borders. Tetrahedron $(C, V)$ is the tetrahedral graph with its six edges labeled by the particle types corresponding to the faces and edges adjoining $V$.

Let $v=\#$ vertices, $f=\#$ faces, $e=\#$ edges. When applied to the net which consists of a large chunk of hexagonal lattice, the shadow method reduces to the following: the region containing the point at infinity is black, and the sum over colorings becomes a sum over colorings in which there are no adjacent black hexagons (and no black hexagons adjacent to the black outside region). Let us first compute the weight of the all white coloring. We ignore boundary effects. We have first of all $v=2 f$ and $e=3 f$. Each edge contributes $\Theta^{-1}=\tau^{-3 / 2}$, each vertex Tetrahedron $=-\tau$, and each face the quantum dimension $\tau$. The total weight of the all white coloring is thus

$$
\left(\tau^{-3 / 2}\right)^{e}(-\tau)^{v}(\tau)^{f}=\tau^{-3 f / 2}
$$

Now suppose we have a configuration with some black hexagons. Its weight is just that of the all white configuration multiplied by appropriate ratios of theta symbols, tetrahedron symbols, and quantum dimensions. More specifically, for each white hexagon that 
one turns into a black hexagon, one must multiply the edge contribution by $\left(\tau / \tau^{3 / 2}\right)^{-6}$, the vertex contribution by $\left(\tau^{3 / 2} /(-\tau)\right)^{6}$, and the face contribution by $\tau^{-1}$. The product of these is $\tau^{5}$, so that the weight of each such coloring is just $\tau^{5 \# \text { (black hexagons) times }}$ the weight of the all-white coloring. The sum over colorings now just yields the critical Hard Hexagon model, whose free energy per vertex was obtained by Baxter [12] (Eq. 10). We thus obtain

$$
G_{\tau}=\left(\tau^{-3 / 2} \kappa_{c}\right)^{f}
$$

where

$$
\kappa_{c}=\left(\frac{27(25+11 \sqrt{5})}{250}\right)^{1 / 2} .
$$

From (22) we surmise

$$
\chi(\tau+1)=G_{\tau} \tau^{f-5 / 4 v}=\left(\tau^{-3} \kappa_{c}\right)^{f} .
$$

We note that this is sharper than Tutte's bound [9] of const. $\tau^{-f}$ on $\chi(\tau+1)$ :

$$
0.546^{f}<0.618^{f} \text {. }
$$

Tutte's bound is of course more general in that it applies to any net configuration on the sphere (whose regions are simply connected and don't border on themselves). For completeness, we note from (26) and (A1) that $c_{0}=\tau^{-3 / 2} \kappa_{c}$.

\section{References}

1. Levin, M., Wen, X.G.: Phys. Rev. B. 71, 045110 (2005)

2. Fendley, P., Fradkin, E.: Phys. Rev. B. 72, 024412 (2005)

3. Moore, G., Read, N.: Nucl. Phys. B 360, 362 (1991)

4. Morf, R.H.: Phys. Rev. Lett. 80, 1505 (1998)

5. Rezayi, E.H., Haldane, F.D.M.: Phys. Rev. Lett. 84, 4685 (2000)

6. Moessner, R., Sondhi, S.L.: Phys. Rev. Lett 86, 1881 (2001); Nayak, C., Shtengel, K.: Phys. Rev. B 64, 064422 (2001); Balents, L. et al.: Phys. Rev. B 65, 224412 (2002); Ioffe, L.B. et al.: Nature 415, 503 (2002); Motrunich, O.I., Senthil, T.: Phys. Rev. Lett. 89, 277004 (2002)

7. Freedman, M., Nayak, C., Shtengel, K.: http://arXiv.org/list/cond-mat/0309120, 2003; Freedman, M., Nayak, C., Shtengel, K.: Phys. Rev. Lett. 94, 066401 (2005); Freedman, M., Nayak, C., Shtengel, K.: Phys. Rev. Lett. 94, 147205 (2005)

8. Chayes, J.T., Chayes, L., Kivelson, S.A.: Commun. Math. Phys. 123, 53 (1989)

9. Tutte, W.T.: On Chromatic Polynomials and the Golden Ratio. J. Combin. Th., Ser. B 9, 289-296 (1970)

10. Cardy, J.: In: Les Houches 1988, Fields, Strings and Critical Phenomena. Brezin, E., Zinn-Justin, J. (eds.) London: Elsevier, 1989

11. Saleur, H.: Nucl. Phys. B 360, 219 (1991); Pasquier, V., Saleur, H.: Nucl. Phys. B, 330, 523 (1990)

12. Baxter, R.: J. Phys. A: Math. Gen. 13, L61-L70 (1980)

13. Freedman, M., Larsen, M., Wang, Z.: http://arXiv.org/list/quant-ph/0001108, 2000

14. Levin, M., Wen, X.G.: Phys. Rev. Lett. 96, 110405 (2006)

15. Witten, E.: Commun. Math. Phys. 121, 351 (1989)

16. Turaev, V.G.: Quantum Invariants of Knots and 3-Manifolds. Berlin-New York: Walter de Gruyter, 1994

17. Fortuin, C.M., Kasteleyn, P.W.: Physica 57, 536 (1972)

18. Kitaev, A.: Ann. Phys. 303, 2 (2003)

19. Trebst, S., et al.: Phys. Rev. Lett. 98, 070602 (2007)

20. Kauffman, L.H., Lins, S.L.: Temperley-Lieb Recoupling Theory and Invariants of 3-Manifolds. Princeton, NJ: Princeton, University Press, 1994 